\title{
Les Chironomidés (Diptera) de quelques "lagunas" de haute altitude de la Sierra Nevada (Granada, Espagne)
}

\author{
H. Laville 1 \\ A. Vilchez-Quero ${ }^{2}$
}

Mots clés : Diptera, Chironomidae, taxonomie, eaux stagnantes, faunistique, écologie, massif montagneux.

13 espèces identifiées dans 24 * lagunas * de très haute altitude $(2700-3200 \mathrm{~m})$ de la Sierra Nevada portent à 35 le nombre de Chironomidés actuellement recensés dans ce massif montagneux du Sud de l'Espagne. 4 espèces sont nouvelles pour la Péninsule Ibérique. L'exuvie nymphale de Micropsectra coracina (K.) est décrite.

The chironomids (Diptera) of several high altitude * lagunas * of the Sierra Nevada (Granada. Spain).

Keywords : Diptera, Chironomidae, taxonomy, stagnant waters, faunistic, ecology, mountain area.

Thirteen species, identified from 24 " lagunas * at very high altitude $(2700-3200 \mathrm{~m})$ in the Sierra Nevada, increases to 35 the number of chironomid species recorded in the mountain area of Southern Spain. Four species a re new for the Iberian peninsula. The pupal exuviae of Micropsectra coracina $(\mathrm{K}$.$) is described.$

\section{1. - Introduction}

La Sier ra Nevada est le massif montagneux avec des altitudes supérieures à 3000 mètres (Mulhacen $3481 \mathrm{~m}$ : Veleta $3398 \mathrm{~m}$ ) le plus méridional d'Europe.

Sa situation géographique détermine une forte influence méditerranéenne qui se combine avec les particularités propres aux écosystèmes de haute montagne. Elle présente une aire de contact intéressante entre plusieurs régions biogéographiques comme le Sud de l'Europe et l'Afrique du Nord.

De nombreuses « lagunas " situées dans la zone de la "Tundra" à des altitudes supérieures à $2700 \mathrm{~m}$ et regroupant différents types d'eaux stagnantes - lacs, mares, sources - offrent un intérêt

1. Laboratoire d'Hydrobiologie, UA 695 C.N.R.S., Université Paul Sabatier, 118, route de Narbonne, 31062 Toulouse Cedex, France.

2. Departamento Biologie, Facultad de Ciencias, Universidad de Granada, España. particulier pour l'étude des organismes aquatiques. Jusqu'à présent, des études ont été consacrées aux communautés phytoplanctoniques (Martinez 1977. 1980) et zooplanctoniques (Cruz Pizarro 1981).

Les communautés benthiques de ces milieux stagnants n'avaient fait l'objet d'aucune étude ; seuls les Chironomidés des eaux courantes avaient été prospectés, mais de façon plus ou moins épisodique.

Ainsi, les premières récoltes de Chironomidés dans la Sierra Nevada remontent à Bertrand (1956) qui signale 5 espèces -4 Diamesinae +1 Orthocladiinae -, Fittkau (1962) cite le Tanypodinae Conchapelopia pallidula du rio Paterna (1 200-1 $300 \mathrm{~m}$ : Almeria), également récolté par Bertrand. SerraTosio $(1970,1971)$ identifie 5 autres Diamesinae dont 2 nouveaux pour la science, Diamesa veletensis et Potthastia iberica, qu'il décrit.

Enfin, Laville (1970) y signale 11 especes - 1 Tanypodinae, 8 Orthocladiinae et 2 Chironominae - portant à 22 le nombre des espèces recensées essentiellement dans les parties hautes de ce massif montagneux. 
Nous récapitulons ci-après la liste de ces 22 espèces, remise à jour à partir des récentes révisions taxonomiques, et leurs altitudes de récoltes.

BerTRAND (1956).

Boreoheptagia legeri (G.) (sub B. punctulata (G.), Serra- Tosio 1970).

Diamesa latitarsis (G.).

Diamesa steinböcki (G.).

Pseudodiamesa nivosa (G.) $2690 \mathrm{~m}$.

Eukiefferiella cyanea Th.

FITTKAU 1962.

Conchapelopia pallidula (Mg.) $1200-1300 \mathrm{~m}$.

SERRA-Tosio (1970 et 1971).

Boreoheptagia legeri (G.) 900 m, $2200-2400 \mathrm{~m}$.

Diamesa bertrami Edw. $2400-2550 \mathrm{~m}$.

Diamesa iberica Ser-Tos. $750 \mathrm{~m}$.

Diamesa latitarsis (G.) $2200-2550 \mathrm{~m}$.

Diamesa veletensis Ser-Tos. (sub D. hamaticomis, Serra-Tosio 1970) $200-2500 \mathrm{~m}$.

Diamesa zernyi Edw. $2400-3000 \mathrm{~m}$.

LAVILLE 1970.

Psectrotanypus varius (Fabr.) $700 \mathrm{~m}$.

Chaetocladius melaleucus (Mg.) $2800-3000 \mathrm{~m}$.

Eukiefferiella minor (Verr.) / fittkaui Lehm.

$2800-3000 \mathrm{~m}$.

Eukiefferiella tirolensis G. (sub Eukiefferiella sp.).

Limnophyes minimus (Mg.) $900 \mathrm{~m}$.

Metriocnemus hygropetricus K. $2400 \mathrm{~m}$.

Orthocladius frigidus (Zett.) $2200-2550 \mathrm{~m}$.

Paratrichocladius skirwithensis (Edw.) (sub. Syncricotopus nivalis Fitt.).

Thienemannia gracilis K. $2400 \mathrm{~m}$.

Chironomus? riparius $\mathrm{Mg}$. (sub Ch. thummi thummi K.) $700 \mathrm{~m}$.

Rheotanytarsus nigricauda Fitt. $2400 \mathrm{~m}$.

\section{2. - Description des "lagunas" étudiées}

Ces « lagunas" peuvent être considérées soit comme de petits lacs lorsque leur profondeur dépasse 2 mètres, soit comme de petites nappes d'eau stagnantes - mares permanentes ou temporaires - pour des profondeurs moindres $(0,4-1,5 \mathrm{~m})$. Il s'agit parfois de sources limnocrènes.

Ces "lagunas " sont difficiles à dénombrer avec exactitude, soit à cause des difficultés d'accès, soit parce qu'elles s'assèchent pendant l'été. Selon les auteurs, leur nombre se situe entre 42 (Ferrer 1971) et 50 (Morales Baquero \& al 1982).

Sur les 24 « lagunas » étudiées, 22 sont permanentes et 2 - Gemela Pequena et Laguna 3 - sont temporaires.

Ces " lagunas " situées entre 2700 et $3200 \mathrm{~m}$ d'altitude dans la zone de la "Tundra", peuvent être soit indépendantes les unes des autres comme, par exemple, celles de la Vallée de Lanjaron, soit communiquer entre elles comme celles de la Vallée des "Siete lagunas".

Le tableau I récapitule les principales caractéristiques morphométriques et physico-chimiques de ces "lagunas". Les données présentées correspondent à des valeurs moyennes de rnesures effectuées au cours de l'été 1981 ; elles sont reprises de Morales Baquero \& al (1982). Les paramètres $\mathrm{N}$ et $\mathrm{P}$ considérés comme des "Nutrients "et les teneurs en sels (conductivité) permettent de cataloguer ces "lagunas" comme des milieux oligotrophes.

Pour les températures, les chiffres sont des valeurs moyennes estivales qui ne rendent pas bien compte du régime thermique. Les valeurs moyennes des petites pièces d'eau sont davantage liées aux changements climatiques alors que la masse d'eau des plus grandes tend à les amortir. De façon générale les températures augmentent pendant l'été pour descendre à la fin.

\section{3. - Matériel étudié}

45 échantillons ont été recueillis entre juin et septembre 1981.

Des exuvies nymphales et quelques imagos $\sigma^{*}$ noyées ont été récoltées à l'aide de filets à la surface de l'eau ; d'autres imagos or ont été capturées par des chasses en vol.

\section{4. - Liste des espèces de Chironomidés}

La répartition des espèces recensées dans les 24 " lagunas » est récapitulée sur le tableau II et la figure 1 .

Pour chaque espèce, nous donnons :

- les lieux et les dates de récoltes des imagos ( $(\sigma)$ ou exuvies nymphales (E)

- les citations antérieures dans la Péninsule ibérique. Les espèces nouvelles pour la zone 1 de la $\mathrm{Lim}$ nofauna Europaea sont précédées d'un astérisque. 
- la répartition biogéographique

- l'écologie.

\section{Pseudodiamesa nivosa (G.).}

- Virgen Superior: $\sigma$, E (10.7.81, 25.8.81).

Virgen Media : E (10.7.81); L. Larga : E (20.7.81);

Laguneto: $\mathrm{E}$ (20.7.81).

- Espagne : Pyrénées : Lac Paderne (2 $124 \mathrm{~m}$ ) dans la Maladet ta (Bertrand 1956) Sierra Nevada : Siete Laguna (2690 m) (Bertrand 1956); Monte Rosa (2 900-3 000 m) (Wülker 1959).

- Répartition : Boréoalpine : Islande, Suede, Norvège, Irlande, Alpes, Pyrénées, Sierra Nevada (Serra-

Tosio 1972, 1976).
- Ecologie : Espèce plus montagnarde que Pseudodiamesa branickii (Nov.), elle colonise de préférence les sources (Eucrénal), les lacs de haute montagne et plus accessoirement les cours d'eau alpins (SerraTosio 1976).

Dans la Sierra Nevada toutes les récoltes proviennent de pièces d'eau peu profondes $(<1,2 \mathrm{~m})$.

\section{* Acricotopus lucens (Zett.).}

- Virgen Superior : E (18.9.81).

- Espèce nouvelle pour la Péninsule iberique : c'est actuellement la citation la plus méridionale en Europe.

Tableau I. Principales caractéristiques morphométriques et physico-chimiques (données moyennes) de 24 "lagunas " de la Sierra Nevada.

\begin{tabular}{|c|c|c|c|c|c|c|c|c|c|c|}
\hline Lagunas & $\begin{array}{c}\text { Altitude } \\
\text { m }\end{array}$ & $\begin{array}{l}\text { Dim } \\
\text { L. }\end{array}$ & $\begin{array}{l}\text { nsions } \\
1 .\end{array}$ & $\begin{array}{l}/ \mathrm{m} \\
\text { prof. }\end{array}$ & Temp. & $\begin{array}{l}\text { Conduct. } \\
\mu \mathrm{mho} / \mathrm{cm}\end{array}$ & $\mathrm{pH}$ & $\underset{\mathrm{mg} / 1}{\mathrm{O}_{2}}$ & $\begin{array}{l}\mathrm{NO}_{3}-\mathrm{N} \\
\mu \text { g.at. } .^{-1}\end{array}$ & $\underset{\mu \mathrm{g} \cdot \text { at. } \mathrm{l}^{-\mathrm{H}}}{\mathrm{PO}_{4}-\mathrm{P}}$ \\
\hline \multicolumn{11}{|l|}{ VALLE R. LANJARON } \\
\hline Cuadrada & $2700 \cdot 2800$ & 30 & 18 & - & 15.4 & 9.0 & 5.9 & 6.0 & 0.17 & 0.65 \\
\hline Penon Colorado & $3000-3100$ & 15 & 9 & 0,5 & 14.9 & 14.6 & 6.9 & 7.8 & 0.45 & 1.20 \\
\hline Lanjarón & $3000-3100$ & 40 & 25 & - & 15.6 & 12.5 & 6.7 & 6.6 & 0.28 & 0.00 \\
\hline Lanjarón 1 & $3000-3100$ & 65 & 25 & 3 & 16.1 & 11.5 & 6.5 & 6.2 & 0.42 & 0.40 \\
\hline \multicolumn{11}{|l|}{ VALLEE R. SECO } \\
\hline Rio Seco Superior & $3000-3100$ & 20 & 15 & 2 & 12.5 & 24.1 & 6.5 & 7.4 & 0.30 & 0.26 \\
\hline Rio Seco & $3000-3100$ & 80 & 30 & 2.5 & 13.2 & 14.5 & 6.5 & 7.1 & 0.33 & 0.43 \\
\hline Aguas Verdes & $3000-3100$ & 50 & 30 & 1.5 & 10.5 & 33.2 & 6.5 & 7.9 & 0.19 & 0.25 \\
\hline \multicolumn{11}{|c|}{ BORREGUILES DE DILAR } \\
\hline Virgen Superior & $2900-3000$ & 50 & 10 & 1.1 & 8.8 & 75.8 & 7.1 & 8.6 & 0.10 & 0.33 \\
\hline Virgen Media & $2900-3000$ & 20 & 10 & 0.5 & 14.8 & 59.8 & 6.6 & 7.5 & 0.76 & 0.53 \\
\hline Virgen Inferior & $2900-3000$ & 25 & 8 & 0,4 & 17.7 & 38.6 & 6.4 & 6.7 & 0.17 & 0.53 \\
\hline Dilar 1 & $2700-2800$ & 17 & 8 & 0,4 & 10.3 & 53.9 & 6.8 & 8.0 & 0.20 & 0.00 \\
\hline Dilar 2 & $2700-2800$ & 15 & 8 & 0,2 & 10.4 & 74.5 & 6.8 & 9.2 & 0.80 & 0.17 \\
\hline \multicolumn{11}{|c|}{ VALLEE R. MULHACEN } \\
\hline Majano & $2900-3000$ & 80 & 50 & 1,2 & 18.8 & 47.1 & 7.4 & 7.8 & 0.36 & 0.00 \\
\hline Gemela Grande & $3000-3100$ & 25 & 18 & 0.5 & 21.6 & 29.5 & 7.2 & 6.7 & 0.14 & 0.20 \\
\hline Gemela Pequena (T.) & $3000-3100$ & 18 & 15 & 0.5 & 22.6 & 27.5 & 7.2 & 7.3 & 0.22 & 0.43 \\
\hline Penon Negro & $2800-2900$ & 100 & 80 & 2 & 13.1 & 26.8 & 6.8 & 7.9 & 1.15 & 0.00 \\
\hline \multicolumn{11}{|l|}{ VALLE 7 LAGUNAS } \\
\hline Laguna 2 & $2900-3100$ & 65 & 60 & 2,5 & 12.7 & 22.8 & 6.4 & 7.4 & 0.65 & 0.12 \\
\hline Laguna 3 (T.) & $2900 \cdot 3100$ & 75 & 75 & 2.5 & 11.3 & 20.9 & 6.4 & 7.5 & 10.60 & 0.00 \\
\hline Laguna 4 & $2900-3100$ & 40 & 20 & 3 & 12.5 & 20.8 & 6.4 & 7.8 & 0.52 & 0.20 \\
\hline Laguna 5 & $2900-3100$ & 30 & 20 & 1 & 18.7 & 38.7 & 6.8 & 7.4 & 0.37 & 0.27 \\
\hline Laguna 7 & $2900-3100$ & 150 & 40 & 1 & 14.8 & 27.7 & 7.0 & 7.8 & 4.60 & 0.00 \\
\hline Mosca & $3100-3200$ & 75 & 35 & - & 10.0 & 33.9 & 6.8 & 7.8 & 0.35 & 0.00 \\
\hline \multicolumn{11}{|c|}{ VALLE R. VALDEINFIERNO } \\
\hline Larga & $2700-2800$ & 200 & 40 & - & 15.1 & 23.8 & 5.6 & 7.1 & 1.15 & 0.00 \\
\hline Laguneto & $2800-2900$ & 25 & 12 & 1.2 & 7.9 & 38.7 & 6.2 & 8.0 & 6.65 & 0.00 \\
\hline
\end{tabular}


Tableau II. Répartition des Chironomidés dans 24 "lagunas » de la Sierra Nevada.

$x$ : exuvies; ; imagos o

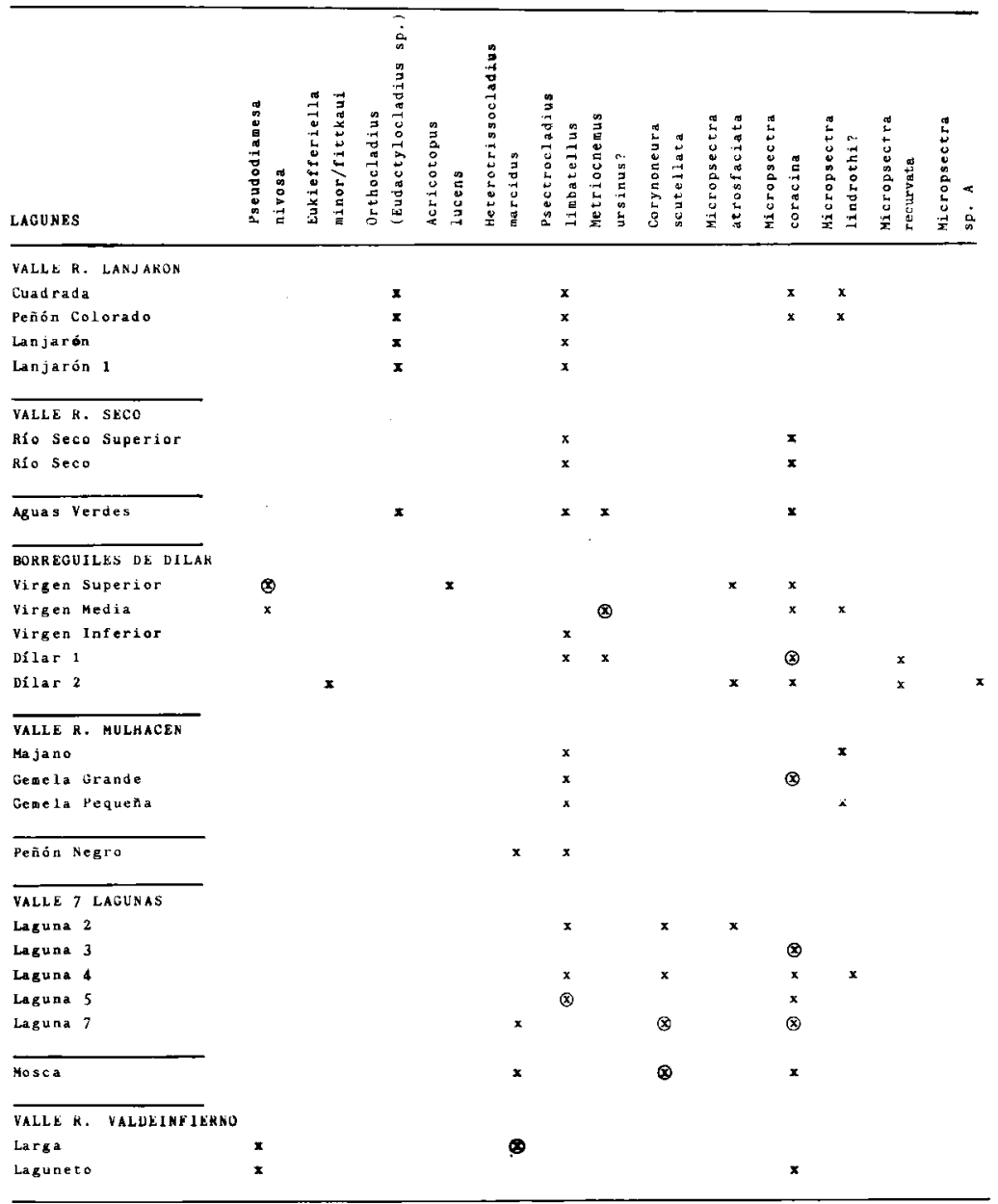




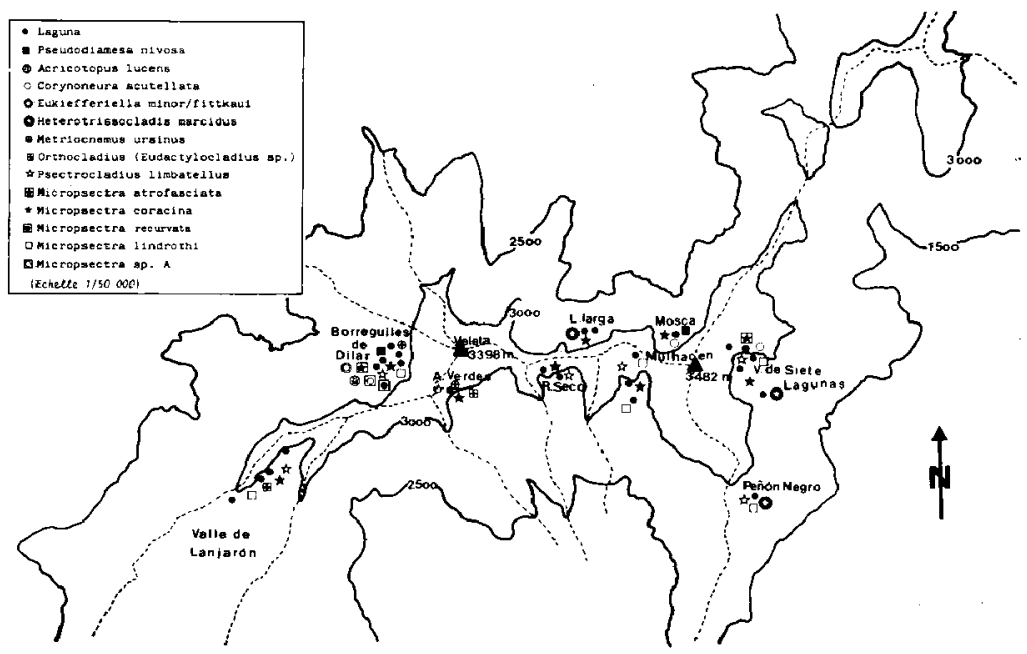

Fig. 1 : Distribution des Chironomidés dans les "lagunas" de haute altitude (2 700-3 $200 \mathrm{~m})$ de la Sierra Nevada.

- Répartition : Europe.

- Ecologie : les larves de $A$. lucens colonisent les eaux stagnantes de différents types, surtout les petites pièces d'eau (Brundin 1956, Hirvenoja 1973).

\section{Corynoneura scutellata Winn.}

- Laguna 2: E (16.7.81); Laguna 4: E (4.7.81); Laguna $7:$ E, $\sigma(21.7 .81)$; Mosca E, $\sigma(21.7 .81)$. - Espagne: Pyrénées: Lac Llong (2000 m) (Prat 1981).

- Répartition : Europe, Groenland.

- Ecologie : Espèce fréquente dans les lacs de montagne - Alpes, Forêt-Noire, Pyrénées - (Schlee 1968). Elle peut coloniser différents types d'eau stagnantes avec un préférendum pour les eaux oligotrophes froides (Laville 1971).

Eukiefferiella minor (Verr.) /ftttkaui Lehm.

- Dilar 2 : E (19.8.81).

- Espagne: E. minor? (1 o) signalé par Laville (1970) du Slope Veleta (2 800-3 000 m) demande confirmation.
- Répartition : E. minor: toute l'Europe ; E. fittkaui : régions montagneuses de l'Europe.

- Ecologie: Dans les Pyrénées ces 2 espèces, impossibles à différencier sur l'exuvie nymphale, seraient vicariantes : $E$. fittkaui caractérise le torrent d'Estaragne ( $>2150 \mathrm{~m}$ ) riche en Diamesinae -alors que $E$. minor colonişe la zone inférieure (2 $150-1100 \mathrm{~m}$ ) plus riche en Eukiefferiella spp. (Laville \& Lavandier 1977, Gazagnes \& Laville 1985). Ces deux Eukiefferiella peuvent être considérés comme rhéophiles, psychrothermes et polyoxybiontes.

\section{Heterotrissocladius marcidus (Walk.).}

- Penón Negro: E (17.7.81); Laguna 7 : E (4.7.81, 16.7.81); La Mosca: E (21.7.81); L. Larga E, o (20.7.81 et 18.8.81).

- Espagne: Pyrénées orientales : Lac Llebreta (1 $600 \mathrm{~m}$ ), Lac de Guits (2000 m) (Prat 1977).

- Répartition : Europe occidentale : des Carpathes, Alpes, Pyrénées jusqu'en Suède, Islande : Canada (Saether 1975). 
- Ecologie: espèce psychrotherme et largement eurytope qui colonise plus particulièrement les zones littorales des lacs de montagne.

\section{* Metriocnemus ? ursinus (Holm.).}

- Aguas Verdes : E (14.7.81); Virgen media : E, o (18.9.81); Dilar 1 : E (10.7.81).

- Espèce nouvelle pour l'Espagne.

- Répartition : boréoalpine.

- Ecologie : la larve vit dans les mousses humides autour de sources froides. Les récoltes de la Sierra Nevada sont situées entre $2700 \mathrm{~m}$ et $3100 \mathrm{~m}$.

\section{Orthocladius (Eudactylocladius) sp.}

- L. Cuadrada: E (7.7.81); Penon Colorado: E (7.7.81); Lanjaron : E (7.7.81); Lanjaron 1 : E (7.7.81); Aguas Verdes : $\mathrm{E}(\mathbf{1 4 . 7 . 8 1 )}$. Une seule espèce du sousgenre Eudactylocladius est signalée de la zone 1 de la Limnofauna (Fittkau \& Reiss 1978) O. fuscimanus (K.) sub. O. bipunctellus (Zett.) n. syn (Cranston 1984). Elle a été récemment rencontrée en Espagne dans les bassins de l'Alhambra et du Generalife de Granada (Vilchez \& Casas, sous presse).

Psectrocladius (s. str.) limbatellus (Holm.) $=$ Ps. edwardsi Br. (Langton 1984).

- L. Cuadrada: E (7.7.81); Penon Colorado: E (7.7.81); Lanjaron : E (7.7.81); Lanjaron 1 : E (7.7.81); Rio Seco Superior: E (14.7.81); Rio Seco: E (14.7.81); Aguas Verdes : E (14.7.81); Dilar 1: E (25.8.81); Dilar 2 : E (25.8.81); Majano : E (30.6.81); Gemela Grande: E $(30.6 .81,7.7 .81)$; Gemela Pequena : E (30.6.81); Penon Negro: E (17.7.81); Laguna 2 : $\mathrm{E}(10.7 .81) ; 16.7 .81)$; Laguna $5: \mathrm{E}, \sigma$ (4.7.81, 16.7.81).

- Espagne : Centre : Almonacid de Toledo(Wülker 1956): Sud : Guadalmena, réservoir sur affluent du Guadalquivir (Prat 1978).

- Répartition: Espèce du genre Psectrocladius avec la plus vaste répartition: connue depuis le Groenland jusqu'aux confins du Sahara ; au Maroc, signalée, du Lac Tamdha $(2800 \mathrm{~m})$ dans le HautAtlas par Wülker (loc. cit.).

- Ecologie: Comme la plupart des espèces du genre, elle colonise tous les types d'eaux stagnantes : lacs, étangs, réservoirs. Les nombreuses récoltes en zone arctique et subarctique et dans les massifs montagneux d'Europe et du Maroc témoignent d'une probable psychrothermie de cette espèce.

\section{Micropsectra atrofasciata $\mathbf{K}$.}

- Virgen Superior: E (18.9.81); Laguna 2: E (10.7.81, 16.7.81).

- Espagne : Catalogne: Rio Ter (Prat 1977); Rio Llobregat (Rieradevall 1985). Bassins de l'Alhambra et du Generalife (Granada) (Vilchez \& Casas, sous presse).

- Ecologie: Espèce eurytherme et relativement eurytope des eaux courantes (Säwedal 1982).

\section{* Micropsectra coracina (K.).}

Lauterbomia coracina $\mathrm{n}$. syn. (Säwedal 1982).

- L. Cuadrada : E (7.7.81); Penon Colorado: E (7.7.81, 28.8.81); Rio Seco Superior : E (14.7.81); Rio seco: E (14.7.81); Aguas Verdes : E (14.7.81); Virgen Superior : E, $O(25.8 .81,18.9 .81)$; Virgen Media E (18.9.81); Dilar 1 : E (25.8.81); Dilar 2: E (18.9.81); Gemela Grande: E, or (2.9.81); Laguna 5: E (16.7.81); Laguna $7: \mathrm{E}(4.7 .81)$; La Mosca : E, $\odot$ (21.7.84); Laguneto: E (20.7.81).

- Espece nouvelle pour l'Espagne.

- Répartition : Europe.

- Ecologie : Espèce psychrotherme qui, dans les régions arctiques, peut coloniser les mares ou les lacs peu profonds mais qui colonise plutòt la zone profonde, bien oxygénée des lacs oligotrophes d'Europe moyenne (Brundin 1949). Dans les Pyrénées, elle a été récoltée en vol près des lacs réservoirs d'Aumar (2 192 m, p. 24 m) et d'Aubert (2 148 m, p. $55 \mathrm{~m}$ ) (Laville 1971), près des sources tributaires du Lac d'Orédon (1850-2050 m) (Laville \& Lavandier 1977) et du lac d'Oô (1 $496 \mathrm{~m}$ ) (Laville 1966).

Nous donnons une nouvelle description de l'exuvie nymphale plus conforme à la taxonomie du genre Micropsectra récemment précisée par Säwedal (1982). La corne thoracique et l'ornementation abdominale sont tout à fait typiques du genre Micropsectra et justifient totalement la mise en synonymie du genre Lauterbornia par Säwedal.

Imago $\sigma$ : Hypopyge: Brundin (1949 : fig. 235 , p. 848)

Nymphe: Bause (1913: p. 103-104; fig. 101 et 102) Zavrel (1926 : p. 206-207)

Bause (1913) figure l'ornementation et la chaetotaxie abdominales (fig. 101) et un renflement ovoïde pédonculé, en guise de corne thoracique (fig. 102) de la nymphe. 
Quelques différences s'observent par rapport aux spécimens de la Sierra Nevada, notamment: absence de courtes soies LS à l'angle postérieur des tergites IV et $V$, et présence d'un chagrin plus large sur les tergites VI et VII, et sur la moitié antérieure du tergite VIII.

Zavrel (1926) donne une description succincte de la nymphe $(\mathrm{l}=7.8 \mathrm{~mm})$, mais signale, chez un spécimen du Wigry.See, la présence d'une longue corne thoracique (1 $500 \mu$ ) garnie de nombreuses soies et dont le renflement chitinisé constitue la base ; $i$ souligne justement que la partie tubuleuse de la corne tombe (par autotomie?) facilement et régulièrement.

Sur les 30 exuvies observées de la Sierra Nevada, 9 seulement possédaient des cornes thoraciques intactes.

Nouvelle description de la nymphe

Longueur : 4,8 - 6,1 $\mathrm{mm}(\mathbf{M}=5,3)(\mathrm{n}=30)$

Coloration : Exuvie transparente sauf le thorax, les fourreaux antennaires, la partie fe + ti des fourreaux de $\mathbf{P}_{1}$, les bords des fourreaux alaires, les bords latéraux du tergite VII, le bord anal et les bords latéraux du tergite VIII, les fourreaux gonopodiaux, brun clair à brun plus foncé.

\section{Céphalothorax}

Tubercules frontaux très courts ou absents, terminés par une soie frontale longue de 150 à $180 \mu$ (fig. 3c). Base des fourreaux antennaires avec une rangée circulaire de fines spinules.

Corne thoracique longue de 700 à $1000 \mu$ r assez tubuleuse jusqu'en son extrémité, avec toute la surface garnic de soies atteignant $1 / 5$ à $1 / 3$ de sa longueur (fig. 3 b).

Suture thoracique avec un léger renflement médian garni de fins granules.

Chaetotaxie du thorax (fig. 2)

De chaque côté, 6 soies thoraciques orales (Oth 1-6).

Oth 1-3 (ou soies précornéales, Pc) de longueurs subégales $(90-100 \mu)$ sont situées à l'avant et légèrement au-dessous de la base de la corne thoracique, avec parfois une petite dent chitinisée proche de leur base.

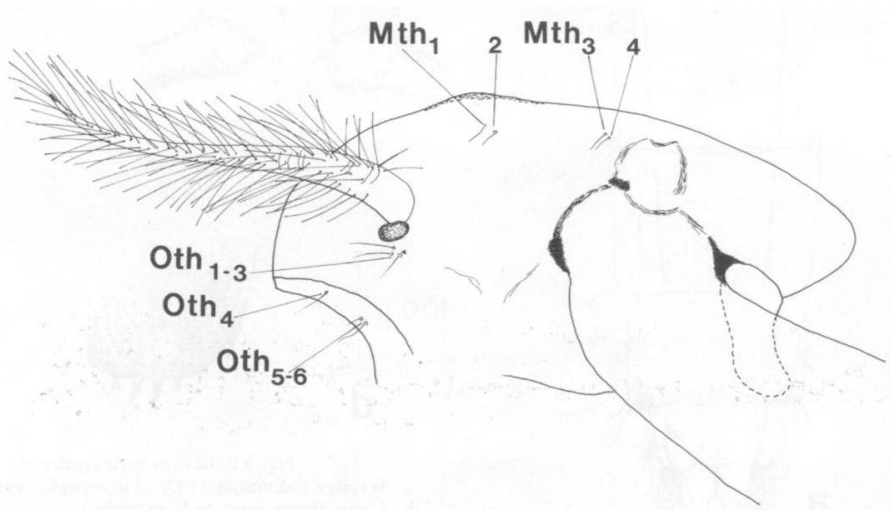

Fig. 2 : Micropsectra coracina: thorax de la nymphe, vue latérale. 

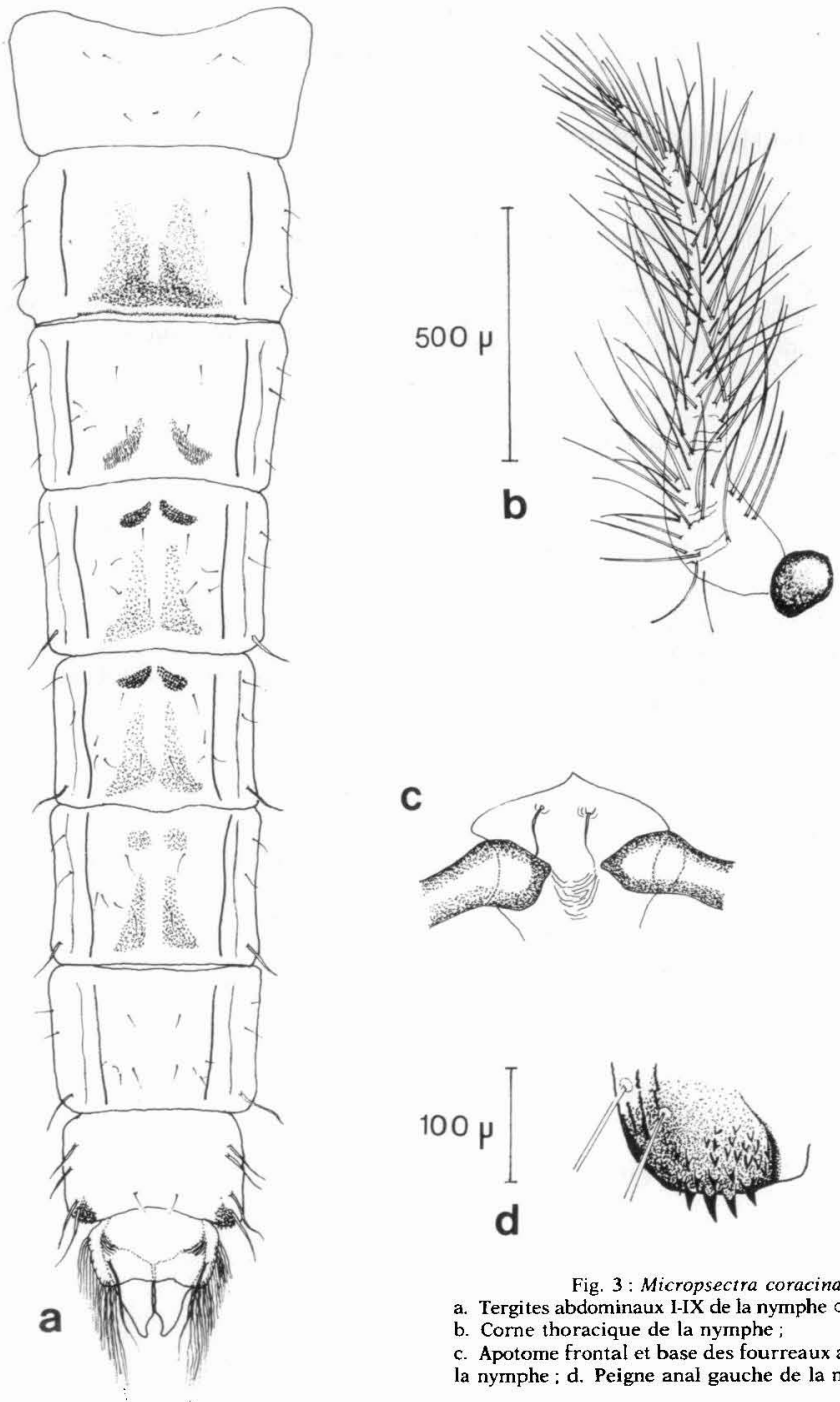

Fig. 3 : Micropsectra coracina:

a. Tergites abdominaux I-IX de la nymphe $\sigma$, vue dorsale ;

b. Corne thoracique de la nymphe ;

c. Apotome frontal et base des fourreaux antennaires de la nymphe: d. Peigne anal gauche de la nymphe. 
Oth 4 - Oth 6 (ou soies antepronatales latérales, LAps) : Oth $4(120 \mu)$ est situee environ aux $2 / 5^{\circ}$, Oth $5\left(30_{\mu}\right)$ et Oth $6(90 \mu)$ très rapprochées au $4 / 5^{\mathrm{e}}$ de la région prothoracique.

4 soies métathoraciques (ou soies dorsocentrales, Dc) longues d'environ $60 \mu$. sont situées en 2 groupes séparés proches de la suture : Mth1 - Mth2 audessous de la partie légèrement renflée et granulée de la suture, Mth 3 - Mth4 au-dessus de la partie antérieure des fourreaux alaires.

Abdomen (fig. 3 a)

Tergite I sans chagrin.

Tergite II avec une rangée de crochets recourbés vers l'avant, qui occupe un peu plus de la moitié de la largeur du segment. Pseudopodes peu apparents sur les pleurites du même segment. Fin chagrin sur deux bandes latérales, séparées par une bande médiane dépourvue de spinules, et se rejoignant à l'arrière le long de la rangée de crochets.

Tergite III avec deux zones postérieures garnies de spinules longues et fines, le reste du tergite en est dépourvu.

Tergite IV et $V$ avec deux champs antérieurs et transversaux de courtes spinules, plus ou moins en forme de croissant, d'où partent deux bandes longitudinales de chagrin plus apparent dans la moitié anale.

Tergite VI avec deux petites zones arrondies antérieures et deux petites zones allongées postérieures très finement chagrinées.

Tergites VII et VIII sans chagrin.

Peignes anaux (fig. 3 d) du tergite VIII avec une vingtaine d'épines chitineuses dont 5-7 distales sont plus longues.

Lobe anal avec une simple rangée de 28-43 ( $\mathrm{M}=$ $33, \mathbf{n}=30$ ) soies filamenteuses, un peu plus courtes dans la moitié an térieure. Face dorsale de chaque lobe avec une longue soie filamenteuse.

\section{Chaetotaxie de l'abdomen}

$\begin{array}{cccccccccc}\text { Soies } & \text { I } & \text { II } & \text { III } & \text { IV } & \text { V } & \text { VI } & \text { VII } & \text { VIII } & \text { IX } \\ \text { D } & 3 & 3 & 5 & 5 & 5 & 5 & 5 & 1 & 1 \\ \text { L } & - & 3 & 3 & 2 & 2 & 2 & 2 & - & - \\ \text { LS } & - & - & - & 1 & 1 & 1 & 2 & 4-5 & - \\ \text { V } & - & 4 & 4 & 4 & 4 & 4 & 4 & 1 & -\end{array}$

Micropsectra lindrothi G.

- L. Cuadrada : E (28.8.81); Penon Colorado: E (28.8.81); Virgen Media : E (18.9.81); Majano E
(2.9.81); Gemela Pequena : E (21.9.81); Laguna 4: E (29.9.81).

- Espagne: Sud-Est : Puentes (Prat 1978).

- Répartition : Large distribution en Europe du Nord-Ouest : France, Allemagne, Islande, Suède, Groeland et U.R.S.S. (Säwedal 1976).

- Ecologie : Espèce crénophile, oxybionte et psychrotherme qui affectionne les substrats riches en matière organique; elle peut également coloniser l'Epirhithral (Säwedal 1982). Dans les Pyrénées, elle est fréquente dans la source hélocrène du Lac Vert (2 $100 \mathrm{~m}$ ) très riche en détritus (Laville 1971, sub M. foliata Lav.).

* Micropsectra recurvata $\mathbf{G}$.

-- Dilar 1 : E (10.7.81); Dilar 2: E (10.7.81).

- Espèce nouvelle pour la Péninsule ibérique.

- Répartition : Europe, Groenland

- Ecologie : Espèce psychotherme et crénobionte qui peut également coloniser les faciès lentiques de l'Epirhithral (Säwedal 1982).

\section{Micropsectra sp A.}

- Dilar 2 : E (18.9.81).

Cette exuvie nymphale présente une ornementation des tergites III-V et une chaetotaxie latérale des segments abdominaux IV-VIII du type contracta Reiss; elle en diffère à la fois par l'absence de longs tubercules frontaux (Langton 1984 : pl. 100 e) et par son écologie puisque $M$. contracta est une espèce typique de la zone profonde de plusieurs lacs oligotrophes de la région alpine (Reiss 1965).

\section{5. - Conclusion}

Les 13 espèces recensées dans 24 "lagunas " de très haute altitude de la Sierra Nevada portent à 35 le nombre des Chironomidés actuellement connus de ce massif montagneux. 4 espèces - Acricotopus lucens, Metriocnemus? ursinus, Micropsecira coracina, Micropsectra recurvata - sont nouvelles pour la Péninsule ibérique.

Parmi les 13 espèces nouvellement récoltées, 8 sont des espèces sténothermes d'eau froide : quatre d'entre'elles sont plutôt crénobiontes - Pseudodiamesa nivosa, Metriocnemus? ursinus, Micropsectra lindrothi, Micropsectra recurvata -, les quatre autres sont surtout présentes dans des lacs oligotrophes des régions montagneuses ou boréales - Corynoneura scutellata, Heterotrissocladius marcidus, Psectrocladius limbatellus, Micropsectra coracina. 
C'est dans ce second groupe que se trouvent les deux espèces les plus fréquentes $-P$ s. limbatellus et $M$. coracina- récoltées dans 16 stations différentes. Viennent ensuite, par ordre décroissant, $\boldsymbol{M}$. lindrothi (6 st.), Eudactylocladitus sp (5 st.), Ps. nivosa, C. scutellata, $H$. marcidus (4 st.)

Les autres espèces n'ont été recensées qu'à 1, 2 ou 3 stations.

Dans chaque "laguna ", seulement 1 à 5 especes sont dénombrées. Bien qu'il s'agisse de récoltes ponctuelles (stations échantillonnées une ou deux fois au cours de l'été), cette faible diversité spécifique peut être mise en relation avec la zone altitudinale (2 700-3 $250 \mathrm{~m})$, une des plus hautes prospectées en Europe.

Les conditions vitales, de plus en plus rigoureuses avec l'altitude, contribuent à l'appauvrissement progressif de la faune.

Dans un lac des Alpes de Savoie, le lac de MontCoua, situé dans la même zone altitudinale ( $2672 \mathrm{~m}$ ), Serra-Tosio (1978), sur la base d'une seule récolte estivale, signale seulement 2 espèces de Chironomidés.

Bien qu'il n'existe pas dans la littérature de données sur des milieux directement comparables, le peuplement chironomidien d'un lac oligotrophe des Alpes autrichiennes, le Gossenköllesee (2 $415 \mathrm{~m}$, prof : $9,9 \mathrm{~m}$ ) étudié par Pechlaner \& Zaderer (1985) et, dans les Pyrénées, celui très semblable de la zone littorale $(<$ $7 \mathrm{~m}$ ) du lac de Port-Bielh (2 $385 \mathrm{~m})$ étudié par Capblancq \& Laville (1983) peuvent être confrontés (Tableau III).

Les espèces fondamentales de ces 2 lacs alpin et pyrénéen, sont les mêmes, à l'exception de $M$. coracina. Cette espèce qui n'a pas été récoltée au lac de Port-Bielh existe cependant dans plusieurs lacs réservoirs plus profonds de ce même massif pyrénéen : les lacs d'Aumar, d'Aubert et d'Orédon.
Dans les \& lagunas " de la Sierra Nevada, ni $M$. contracta ni $P$. austriacus n'ont été trouvées. Pour $M$. contracta, cette absence peut être mise en relation avec la faible profondeur $(<3 \mathrm{~m})$ des milieux prospectés puisqu'il s'agit d'une espèce typique de la zone profonde de lacs oligotrophes.

L'absence de $P$. austriacus, à priori, s'explique moins car cette espece, plus eurytherme et plus eurytope, est souvent signalée de petites pièces d'eau stagnante. En Espagne, elle a été signalée d'un lac pyrénéen, le lac Paderne (2 $124 \mathrm{~m}$ ) dans le massif de la Maladetta (Ber. trand 1953) et d'un lac réservoir du Nord-Ouest, le Lago di Sanabria (Prat 1980). Soit, la zone prospectée se situe au-dessus de la limite altitudinale supérieure de l'espèce, soit, les dates d'échantillonnage ne coïncident pas avec sa phénologie.

En conclusion, ce premier inventaire du peuplement Chironomidien de petites pièces d'eau stagnante de la Sierra Nevada, pourrait être élargi au moyen de récoltes tenant compte du cycle des espèces. Cela devrait permettre de compléter l'inventaire des espèces vivant réellement dans ces milieux extrêmes et de préciser certains points de leur écologie.

\section{Travaux cltés}

Bause (E.). 1913. - Die Metamorphose der Gattung Tanytarsus und einiger verwandter Tendipedidenarten. Ein Beitrag zur Systematik der Temdipediden. Arch. Hydrobiol. Suppl. 2 : 1-126.

Bertrand $\left(\mathrm{H}_{+}\right)+1953$. - Diptères Chironomides pyrénéens (2* note), Bull. Soc. ent. Fr. 58: 76-79.

Bertrand $(\mathbf{H}$.) 1956. - Diptères Chironomides pyrénéens et espagnois, Bull. Soc, ent. Fr. 6: : 93-95.

Brundin (L.). 1949. - Chironomiden und andere Bodentiere der südschwedischen Urgebirgsseen. Ein Beitrag zur Kenntnis der bodenfaunistischen Charak terzüge schwedischer oligot ropher Seen. Rep. Inst. Freshwat. Res. Drottningholm $30: 914$ pp.

Brundin (L.). 1956. - Zur Systematik der Orthocladinae (Dipt. Chironomidae), Rep. Inst. Freshwat. Res. Drotiningholm, 37 : 1-185.

Capblancq (J) \& Laville (H.). 1983. - Le lac de Port-Bielh (Pyrénées) : exemple de fonctionnement d'un écosystème lacustre de haute montage : 51-79, In Problemes d'Ecologie : Ecosystèmes limniques. Masson, Paris.

Tableau III. Espèces communes du lac alpin Gossenköllesee (2 $415 \mathrm{~m})$. de la zone littorale $(0.7 \mathrm{~m})$ du lac pyréréen de Port-Bielh $(2385 \mathrm{~m})$ et de a lagunas $n$ de haute altitude $(2700-3200 \mathrm{~m})$ de la Sierra Nevada.

\begin{tabular}{lccc}
\hline & Alpes & Pyrénées & Sierra Nevada \\
\hline Corynoneura scutellata & + & + & + \\
Heterotrissocladius marcidus & + & + & + \\
Micropsectra contracta & + & + & + \\
Micropsectra coracina & + & + & + \\
Paratanytarsus austriacus & + & + & \\
\hline
\end{tabular}


Cranston (P.S.). 1984. - The taxonomy and ecology of Orthociadius (Eudacsylocladius) fuscimanus (Kieffer), a hygropetric chironomid (Diptera), Joumal of Natural History, 18 ; 873-895.

Ferrer (M.), 1971. - Sierra Nevada. Ed. Litografia. Anel. Granada : $635 \mathrm{p}$.

Fittkau (E.J.). 1962 - Die Tanypodinae (Diptera, Chironomidae), Abh. Larvalsyst. Insekten $6: 1-453$.

Fitt kau (E.J.) \& Reiss (F.). 1978. - Chironomidae, In Illies, J. (ed.) Limnofauna Europaea, 2. Aufl : 404-440. G. Fisher, Stuttgart.

Gazagnes (G.) \& Laville (H.7.: 1985 - Etude faunistique des Chironomidés (Diptera) de la Haute Neste d'Aure (Pyrénées centrales) : impact des amenagements hydro-électriques. Annls Limnol. 21 (2): $149-159$.

Hivenoja (M.) : 1973. - Revision der Gattung Cricotopus van der Wulp. und ihrer Verwandten (Diptera, Chironomidae), Ann. Zool. Fennici $10: 1.363$.

Langton (R.H.). 1984 - A key to pupal exuviae of British Chironomidae : 324 p. P.H. Langton (Ed.). March, Cambridgeshire, Great Britain.

Laville (H.) 1966 - Chironomides du Mass if de Néouvielle (Pyrénées centrales), Annls Limmol. 2(1): 203-216.

Laville (H.). 1970 — Some Chironomidae (excl. Diamesinae) from Southern Spain (Insecta. Diptera). Steenstrupia, 1; 21-23.

Laville (H.). 197!. - Recherches sur les Chironomides (Diptera) lacustres du massif de Néouvielle (Hautes-Pyrénées). Première partie : systématique, écologie, phénologie, Arnls Limnol. 7 : 173-332.

Laville (H.) \& Lavandier (P.) 1977 - Les Chironomides (Diptera) d'un torrent pyrénéen de haute montage : l'Estaragne, Annls Limnol. 13: 57-81

Martinez (R.) 1977 - Phytoplankton species, biomass and diversity in lake La Caldera (Sierra Nevada, Granada, Spain), Acta Hydrobiol. 19 (2) : 95-107.

Martinez (R.) 1980 - Seasonal variations of phytoplankton bio mass and photosynsthesis in the high-mountain lake La Caldera (Sierra Nevada, Spain), In Developments in Hydrobiology vol 3: 111-119. M. Dokulil and al (Ed.), The Hague.

Morales Baquero (R.), Cruz Pizarro (L.) \& Canteras Jordana (J.C.). 1982. - Singularidades de los ecosistemas lacustres de alta montana en Sierra Nevada. Actas 1ri. Congr. Nac. Prot. Nat. (sous presse).

Pechlaner (R.) \& Zaderer (P.). 1985 - Interralations between brown trout and chironomids in the alpine lake Gossenkölle see (Tyrol), Verh. Internat. Verein. Limmol. 22 (4) : 2620-2627.

Prat (N.). 1977 - Quironomidos de Cataluna. Graellsia, 31 : 157-185.

Prat (N.). 1978 . - Ecologia y sistematica de Quironomidos Insecta, (Diptera) de los embalses espanoles. Thesis Univ. Barcelona: 359 p.
Prat (N.). 1980. - Ouironomidos de los embalses espanoles (Dip tera) (2* partie), Graellsia, $34: 59-119$.

Prat (N.). 1981. - Quironomidos de Cataluna (2e note), Mediterranea $5: 43-66$.

Reiss (F). 1965. - Micropsectra praecox Meig. und Micropsectra contracta n. sp. (Diptera : Chironomidae), Arch. Hydrobiol. 61 (2) : 228-241.

Rieradevall (M.). 1985. - Ritme diari de la deriva en una estacio del riu Llobregat : Amb especial atencio a les exuvies pupals dels Chironomidae (Dipt.), Tesi de Llicenciatura, Univ. Barcelona : $175 \mathrm{p}$.

Saether (O.A.) 1975. - Nearctic and Palaearctic Heterorrissocladius (Diptera : Chironomidae). Bull. Fish. Res. Bd. Can. 193;67 p.

Säwedal (L.). 1976. - Revision of the notescens-group of the genus Micropsectra Kieffer, 1909 (Diptera: Chironomidae), Ent. scand. $7: 109-144$.

Säwedal (L.). 1982. - Chironomidae, Ent. scand. 13 ; 371-400.

Schlee (D.) 1968. - Vergleichende Merkmalsanalyse zur Morphologie und Phylogenie der Connoneura-Gruppe (Diptera : Chironomidae). Zugleich eine aligemeine Morphologie der Chironomiden-Imago (), Stutt. Beitr. Naturk. 180; 150 pp.

Serra-Tosio (B.). 1970. - Some Diamesinae from southern Spain (Insecta, Diptera, Chironomidae), Steenstrupia : 25-27.

Serra-Tosio (B.). 1971. - Deux Diamesini nouveaux d'Espagne, Traw Lab. Hydrobiol., Grenoble, 62:147-167

Serra-Tosio (B.). 1972. - Ecologie el biogéographie des Diamesini d'Europe (Diptera, Chironomidae), Trav. Lab. Hydobiol. Grenoble, 63 (Année 1971): 5-175.

Serra-Tosio (B.). 1976. - Chironomides des Alpes : le genre Pseudodiamesa (Diptera, Chironomidae), Trav, Sc, P. N. Vanoise, VII : $117-138$.

Serra-Tosio (B.), 1978, - Les Diptères Chironomidés du lac de Mont-Coua (Parc National de la Vanoise), Trav. Sc. P. N. Vanoise, IX : 141-145.

Vilchez (A.) \& Casas (J.). 1986. - Chironomidae (DIptera) from stagnant waters in Granada, Spain. IX Ih International Symposium on Chironomidae, Bergen, July 29-August 3, 1985, (sous presse).

Wülker (W.). 1956. - Zur Kenntnis der Gattung Psectrocladius Kieff. (Dipt., Chironom.). Individuelle Variabilität, Grenzen und Möglichkeiten der Artentrennung, Okologie und Verbreitung, Arch. Hydrobiol. Suppl. 24 : 1.66.

Wülker (W.). 1959. - Diamesarien - Studien (Dipt., Chironomidae) im Hochschwarzwald. Arch. Hydrobiol. Suppl. 24 : 338-360.

Zavrel (J.) - Chironomiden aus Wigry-See. Archwn Hydrobiol. $R y b .1: 195-220$. 